\title{
AN EFFICIENT THREE-TERM CONJUGATE GRADIENT-TYPE ALGORITHM FOR MONOTONE NONLINEAR EQUATIONS
}

\author{
Jamilu Sabi'u and AbDullah ShaH*
}

\begin{abstract}
In this article, we proposed two Conjugate Gradient (CG) parameters using the modified Dai-Liao condition and the descent three-term CG search direction. Both parameters are incorporated with the projection technique for solving large-scale monotone nonlinear equations. Using the Lipschitz and monotone assumptions, the global convergence of methods has been proved. Finally, numerical results are provided to illustrate the robustness of the proposed methods.
\end{abstract}

Mathematics Subject Classification. 90C30, 90C26.

Received April 7, 2020. Accepted June 6, 2020.

\section{INTRODUCTION}

This paper will address the problem

$$
F(x)=0,
$$

where $F: R^{n} \rightarrow R^{n}$ is continuous and monotone function. The nonlinear monotone equations arise in different applications, such as Bregman distances [17], chemical equilibrium systems [22], financial forecasting problems [9] and signal reconstruction problems in compressive sensing [12]. Also, some variational inequality problems can be transformed into a system of monotone nonlinear equations [31].

The Newton method, the quasi-Newton methods and their variants are considered to be efficient methods for solving (1.1) despite the Jacobian inverse or its approximate requirement, see [10,25,33,34]. However, these methods are not suitable for large-scale problems due to the computing and storage of the Jacobian matrix or its approximate for each iteration $[8,20,29]$. Nevertheless, in an effort to solve large-scale monotone nonlinear equations, Zhang and Zhou [30] proposed a spectral approach for (1.1) that combines the two-point gradient method [3] with the projection method [27]. The global convergence of the method [30] is provided using the monotone and Lipschitz continuous assumptions. The spectral method [30] has fairly low computational costs because it does not require the computing and storage of the Jacobian matrix or its approximation at each iteration.

Conjugate gradient methods are considered to be the most reliable numerical methods for solving large-scale problems due to their low memory requirements and good global convergence properties. They are iterative

\footnotetext{
Keywords. Monotone equations, three-term conjugate gradient method, conjugacy condition, descent condition.

Department of Mathematics, COMSATS University Islamabad, Park Road, Islamabad 44000, Pakistan.

*Corresponding author: abdullah_shah@comsats.edu.pk
} 
methods that generate a sequence of solutions

$$
x_{k}=x_{k-1}+\alpha_{k} d_{k-1}
$$

where $x_{k-1}$ is the initial approximation, $\alpha_{k}$ is the positive step length to be determine using an appropriate line search and $d_{k}$ is the CG search direction defined by

$$
d_{k}=-F\left(x_{k}\right), \quad k=0, \quad d_{k}=-F\left(x_{k}\right)+\beta_{k} d_{k-1} \quad k \geq 1,
$$

with $\beta_{k}$ known as the CG update and it is the parameter that characterizes the CG method. The widely known CG update included the Hestenes-Stiefel (HS) formula [15], i.e.,

$$
\beta_{k}^{\mathrm{HS}}=\frac{F_{k}^{T} y_{k-1}}{d_{k-1}^{T} y_{k-1}},
$$

where $F_{k}=F\left(x_{k}\right)$ and $y_{k-1}=F_{k}-F_{k-1}$. However, the numerical performance of the CG methods depended on the appropriate choice of the parameter $\beta_{k}$ [6]. Some important results on the global convergence of the CG methods were reviewed in [14,23]. Dai and Liao [5] incorporated the second-order information into CG method and proposed the following condition

$$
d_{k}^{T} y_{k-1}=-t F_{k}^{T} s_{k-1}
$$

where $t$ is a nonnegative scalar and $s_{k}=x_{k}-x_{k-1}$. However, in order to guarantee that the search direction $d_{k}$ satisfies condition (1.5), they used the CG direction (1.3) into (1.5) and derived the following update

$$
\beta_{k}^{\mathrm{DL}}=\frac{F_{k}^{T}\left(y_{k-1}-t s_{k-1}\right)}{d_{k-1}^{T} y_{k-1}} .
$$

The choice of the parameter $t \geq 0$ is considered by Andrei [1] to be an open problem for nonlinear CG method. This motivated Babaie-Kafaki and Ghanbari [2], and they proposed the following optimal choices for the nonnegative parameter " $t$ " in the $\beta_{k}^{\mathrm{DL}}$ :

$$
t_{k}^{1}=\frac{\left\|y_{k-1}\right\|}{\left\|s_{k-1}\right\|}
$$

and

$$
t_{k}^{2}=\frac{y_{k-1}^{T} s_{k-1}}{\left\|s_{k-1}\right\|^{2}}+\frac{\left\|y_{k-1}\right\|}{\left\|s_{k-1}\right\|}
$$

By several numerical tests, it has proved that the choices $t_{k}^{1}$ and $t_{k}^{2}$ are robust compared to some other CG algorithms. Narushima et al. [24] suggested a descent three-term CG method with the search direction defined by

$$
d_{k}=-F_{k}, \quad k=0, \quad d_{k}=-F_{k}+\beta_{k}\left(F_{k}^{T} p_{k}\right)^{\dagger}\left\{\left(F_{k}^{T} p_{k}\right) d_{k-1}-\left(F_{k}^{T} d_{k-1}\right) p_{k}\right\}, \quad k \geq 1,
$$

where $p_{k}$ is any vector in $R^{n}$, and

$$
A^{\dagger}= \begin{cases}\frac{1}{A}, & \text { if } A \neq 0 \\ 0, & \text { otherwise. }\end{cases}
$$

Furthermore, among the nice properties of the search direction (1.9) is that, it satisfies the following condition

$$
F_{k}^{T} d_{k}=-\left\|F_{k}\right\|^{2}, \quad \forall k \geq 0,
$$

which is independent of the line search and choices of $\beta_{k}$ and $p_{k}$. This shows that the sufficient descent condition is always satisfied for $r=1$, i.e.,

$$
F_{k}^{T} d_{k} \leq-r\left\|F_{k}\right\|^{2}, \quad \forall k \geq 0 .
$$


Many other conjugate gradient methods have been combined with the projection method [27] to solve the large-scale monotone nonlinear equations [4,7,8,19-21, 26, 29].

The main aim of this paper is to develop a robust three-term conjugate gradient method such that the $\beta_{k}$ update can be derived by using the Dai-Liao condition (1.5) with the three-term CG search direction (1.9). The resulting CG update will be used to develop an effective CG method for monotone nonlinear equations using the projection technique.

The rest of the paper is structured as follows: In Section 2, we suggest a new $\beta_{k}$ for the three-term (1.9) method based on the Dai-Liao condition. The convergence of algorithms is shown in Section 3. The numerical results are presented in Section 4. Section 5 is the conclusion.

\section{ThreE-TERM CONJUGATE GRADIENT-TYPE ALGORITHM}

This section will present two formulas for the update $\beta_{k}$ based on the second Dai-Liao condition (1.5) and the three-term CG method (1.9). Now, by incorporating the optimal value (1.7) into the condition (1.5), we get

$$
d_{k}^{T} y_{k-1}=-W_{k}
$$

where $W_{k}=\frac{\left\|y_{k-1}\right\|}{\left\|s_{k-1}\right\|} F_{k}^{T} s_{k-1}$. Now substituting (1.9) into (2.1), we have

$$
\left(-F_{k}+\beta_{k} d_{k-1}-\beta_{k} \frac{F_{k}^{T} d_{k-1}}{F_{k}^{T} p_{k}} p_{k}\right)^{T} y_{k-1}=-W_{k} .
$$

Then the above equation (2.2) can be rewritten as

$$
\beta_{k}\left(d_{k-1}-\frac{F_{k}^{T} d_{k-1}}{F_{k}^{T} p_{k}} p_{k}\right)^{T} y_{k-1}=F_{k}^{T} y_{k-1}-W_{k},
$$

where $y_{k-1}=F_{k}-F_{k-1}$. Now, by using the definition of $y_{k-1}$ in (2.3) gives

$$
\beta_{k}\left(-F_{k-1}^{T} d_{k-1}+\frac{F_{k-1}^{T} p_{k}}{F_{k}^{T} p_{k}} F_{k}^{T} d_{k-1}\right)=F_{k}^{T} y_{k-1}-W_{k} .
$$

Applying condition (1.11) into (2.4) we have

$$
\beta_{k}=\frac{F_{k}^{T} y_{k-1}-W_{k}}{\left\|F_{k-1}\right\|^{2}+Q_{k}}
$$

where $Q_{k}=\frac{F_{k-1}^{T} p_{k}}{F_{k}^{T} p_{k}} F_{k}^{T} d_{k-1}$. We avoided an undefined denominator by modifying (2.5) as

$$
\beta_{k}^{1}=\frac{F_{k}^{T} y_{k-1}-W_{k}}{\left\|F_{k-1}\right\|^{2}+\xi_{k} Q_{k}}
$$

Similarly, using the optimal choice (1.8) into the condition (1.5) and following the same procedure we obtain

$$
\beta_{k}^{2}=\frac{F_{k}^{T} y_{k-1}-H_{k}}{\left\|F_{k-1}\right\|^{2}+\xi_{k} Q_{k}}
$$

where

$$
H=\left(\frac{y_{k-1}^{T} s_{k-1}}{\left\|s_{k-1}\right\|^{2}}+\frac{\left\|y_{k-1}\right\|}{\left\|s_{k-1}\right\|}\right) F_{k}^{T} s_{k-1}
$$


We chose the parameter $\xi_{k}$ such that

$$
\xi_{k}= \begin{cases}\min \left\{1,-\left(1-\xi_{0}\right) \frac{\left\|F_{k-1}\right\|^{2}}{Q_{k}}\right\}, & \text { if } \quad Q_{k}<0 \\ 1, & \text { otherwise }\end{cases}
$$

where $\xi_{0} \in(0,1)$. Kobayashi et al. [18] guaranteed that the denominator of $\beta_{k}^{1}$ and $\beta_{k}^{2}$ always satisfy

$$
\left\|F_{k-1}\right\|^{2}+\xi_{k} Q_{k} \geq \xi_{0}\left\|F_{k-1}\right\|^{2} .
$$

Now, we assume that:

(i) Function $F$ is monotone, that is,

$$
(F(x)-F(y))^{T}(x-y) \geq 0, \quad \forall x, y \in R^{n} .
$$

(ii) Function $F$ is Lipschitz continuous, that is, for some $m>0$ :

$$
\|F(x)-F(y)\| \leq m\|x-y\|, \quad \forall x, y \in R^{n} .
$$

Furthermore, we adopted the concept of Solodov and Svaiter [27] to let the next iterate

$$
x_{k+1}=x_{k}-\frac{F\left(w_{k}\right)^{T}\left(x_{k}-w_{k}\right)}{\left\|F\left(w_{k}\right)\right\|^{2}} F\left(w_{k}\right),
$$

where $w_{k}=x_{k}+\alpha_{k} d_{k}$. Finally, the proposed algorithm is as follows:

Algorithm 2.1 (Efficient three-term CG (ETCG)).

Step 0. Select the starting point $x_{0} \in R^{n}$, and initialize the constants $\gamma \in(0,1), \epsilon, \tau, \delta \geq 0$. Set $k=0$ and $p_{k}=F_{k}$.

Step 1. If $\left\|F_{k}\right\| \leq \epsilon$, stop, if not go to Step 2 .

Step 2. Calculate the search direction

$$
d_{k}=-F_{k}, \quad k=0, \quad d_{k}=-F_{k}+\beta_{k}^{1}\left(F_{k}^{T} p_{k}\right)^{\dagger}\left\{\left(F_{k}^{T} p_{k}\right) d_{k-1}-\left(F_{k}^{T} d_{k-1}\right) p_{k}\right\}, \quad k \geq 1,
$$

or

$$
d_{k}=-F_{k}, \quad k=0, \quad d_{k}=-F_{k}+\beta_{k}^{2}\left(F_{k}^{T} p_{k}\right)^{\dagger}\left\{\left(F_{k}^{T} p_{k}\right) d_{k-1}-\left(F_{k}^{T} d_{k-1}\right) p_{k}\right\}, \quad k \geq 1 .
$$

Step 3. Determine $\alpha_{k}=\max \left\{\tau \gamma^{j}: j=0,1,2, \ldots\right\}$ such that

$$
-F\left(x_{k}+\alpha_{k} d_{k}\right)^{T} d_{k} \geq \delta \alpha_{k}\left\|F\left(x_{k}+\alpha_{k} d_{k}\right)\right\|\left\|d_{k}\right\|^{2} .
$$

Step 4. Let $w_{k}=x_{k}+\alpha_{k} d_{k}$, if $\left\|F\left(w_{k}\right)\right\|=0$ stop, otherwise go to Step 5 .

Step 5. Compute the projection of $x_{k+1}$ using (2.13).

Step 6. Set $k=k+1$ and go to Step 1 .

\section{Convergence analysis}

This section will provide the global convergence of Algorithms (2.1) using the monotonicity and Lipschitz assumptions. Since the search direction (1.9) satisfied the sufficient descent condition irrespective of the choice $\beta_{k}$, we proceed with our proof as follows 
Lemma 3.1 ([27]). Assume that $F$ is monotone and $x, w \in R^{n}$ satisfy $F(y)^{T}(x-y)>0$. Let

$$
x^{+}=x-\frac{F(w)^{T}(x-w)}{\|F(w)\|^{2}} F(w) .
$$

For any $x^{*} \in R^{n}$ such that $F\left(x^{*}\right)=0$,

$$
\left\|x^{+}-x^{*}\right\|^{2} \leq\left\|x-x^{*}\right\|^{2}-\left\|x^{+}-x\right\|^{2} .
$$

Lemma 3.2. Let $F$ be monotone and Lipschitz continuous and $\left\{x_{k}\right\}$ be generated by the Algorithm 2.1. Assume that the solution set of equation (1.1) is nonempty, for any $x^{*}$ such that $F\left(x^{*}\right)=0$, we have

$$
\left\|x_{k+1}-x^{*}\right\|^{2} \leq\left\|x_{k}-x^{*}\right\|^{2}-\left\|x_{k+1}-x_{k}\right\|^{2} .
$$

In fact, $\left\{x_{k}\right\}$ is bounded. This also holds that either $\left\{x_{k}\right\}$ is finite and the last iterate is a solution, or the sequence is infinite and $\lim _{k \rightarrow \infty}\left\|x_{k+1}-x_{k}\right\|=0$. In addition, $\left\{x_{k}\right\}$ converges to some $x^{*}$ such that $F\left(x^{*}\right)=0$.

Proof. First of all, if the Algorithm 2.1 ends at some $k$ iteration, then $d_{k}=0$, and we get $F\left(x_{k}\right)=0$, which means that $x_{k}$ is the solution. Now suppose $d_{k} \neq 0$ for all $\mathrm{k}$, and then an infinite $\left\{x_{k}\right\}$ is generated. It is obvious from equation (2.14) that

$$
F\left(w_{k}\right)^{T}\left(x_{k}-w_{k}\right)=-\alpha_{k} F\left(w_{k}\right)^{T} d_{k} \geq \delta \alpha_{k}^{2}\left\|F\left(w_{k}\right)\right\|\left\|d_{k}\right\|^{2}>0 .
$$

Let $x^{*}$ be any point such that $F\left(x^{*}\right)=0$. By (2.13), (3.4) and Lemma 3.1, we get

$$
\left\|x_{k+1}-x^{*}\right\|^{2} \leq\left\|x_{k}-x^{*}\right\|^{2}-\left\|x_{k+1}-x_{k}\right\|^{2} .
$$

Therefore the sequence $\left\{\left\|x_{k}-x^{*}\right\|\right\}$ is non-increasing and convergent, hence the sequence $\left\{x_{k}\right\}$ is bounded, and also

$$
\lim _{k \rightarrow \infty}\left\|x_{k+1}-x_{k}\right\|=0
$$

Now, from (2.13) and (3.4) we have

$$
\left\|x_{k+1}-x_{k}\right\|=\frac{\left|F\left(w_{k}\right)^{T}\left(x_{k}-w_{k}\right)\right|}{\left\|F\left(w_{k}\right)\right\|} \geq \delta \alpha_{k}^{2}\left\|d_{k}\right\|^{2} .
$$

Hence, from the inequality (3.7) and (3.6) we have

$$
\lim _{k \rightarrow \infty} \alpha_{k}\left\|d_{k}\right\|=0
$$

From the continuity of $F$ and the boundedness of $\left\{x_{k}\right\}$, it is clear that $\left\{x_{k}\right\}$ has some accumulation point $\hat{x}$ such that $F(\hat{x})=0$. Also by choosing $x^{*}=\hat{x}$ in (3.5), since $x^{*}$ is arbitrary, then the sequence $\left\{\left\|x_{k}-\hat{x}\right\|\right\}$ converges. Hence, $\left\{x_{k}\right\}$ converges to $\hat{x}$.

It follows from assumption (ii) that there is a positive constant $\kappa>0$ such that

$$
\left\|F\left(x_{k}\right)\right\| \leq \kappa, \quad \forall k \geq 0 .
$$




\section{Remarks}

(1) Using the definition of $y_{k}$ and the Lipschitz continuity of $F$, we have

$$
\left\|y_{k}\right\|=\left\|F\left(x_{k+1}\right)-F\left(x_{k}\right)\right\| \leq m\left\|x_{k+1}-x_{k}\right\| .
$$

(2) Also by using the definition of $y_{k}, s_{k}$ and the Lipschitz continuity assumption on $F$, we get

$$
\begin{aligned}
\left|y_{k}^{T} s_{k}\right| & =\left|\left(F\left(x_{k+1}\right)-F\left(x_{k}\right)\right)^{T}\left(x_{k+1}-x_{k}\right)\right| \\
& \leq\left\|F_{k+1}-F_{k}\right\|\left\|x_{k+1}-x_{k}\right\|=m\left\|x_{k+1}-x_{k}\right\|^{2} .
\end{aligned}
$$

It follow from (3.9) to (3.11) that

$$
\left|W_{k}\right|=\frac{\left\|y_{k-1}\right\|}{\left\|s_{k-1}\right\|}\left|F_{k}^{T} s_{k-1}\right| \leq \frac{\left\|y_{k-1}\right\|}{\left\|s_{k-1}\right\|}\left\|F_{k}\right\|\left\|s_{k-1}\right\| \leq m \kappa\left\|x_{k}-x_{k-1}\right\|,
$$

and

$$
\begin{aligned}
\left|H_{k}\right| & =\left|\left(\frac{y_{k-1}^{T} s_{k-1}}{\left\|s_{k-1}\right\|^{2}}+\frac{\left\|y_{k-1}\right\|}{\left\|s_{k-1}\right\|}\right) F_{k}^{T} s_{k-1}\right| \\
& \leq\left(\frac{\left\|y_{k-1}\right\|\left\|s_{k-1}\right\|}{\left\|s_{k-1}\right\|^{2}}+\frac{\left\|y_{k-1}\right\|}{\left\|s_{k-1}\right\|}\right)\left\|F_{k}\right\|\left\|s_{k-1}\right\| \\
& \leq 2 m \kappa\left\|x_{k}-x_{k-1}\right\| .
\end{aligned}
$$

Lemma 3.3. Let our assumptions be maintained, and $\left\{x_{k}\right\}$ be generated by our Algorithm 2.1. If there exists a constant $\theta>0$ such that $\left\|F_{k}\right\| \geq \theta$, then there exist $M>0$ such that

$$
\left\|d_{k}\right\| \leq M, \quad \forall k .
$$

Proof. Considering the choices of $p_{k}=F_{k}, \beta_{k}^{1}$ and $\beta_{k}^{2}$, then from (2.10), (3.9), (3.12), (3.13) and the definition of our direction, we have

$$
\begin{aligned}
\left\|d_{k}\right\| & =\left\|-F_{k}+\beta_{k}^{1} d_{k-1}-\beta_{k}^{1} \frac{F_{k}^{T} d_{k-1}}{F_{k}^{T} F_{k}} F_{k}\right\| \\
& \leq\left\|F_{k}\right\|+\frac{\left\|F_{k}\right\|\left\|y_{k-1}\right\|+\left|W_{k}\right|}{\xi_{0}\left\|F_{k-1}\right\|^{2}}\left\|d_{k-1}\right\|+\frac{\left\|F_{k}\right\|\left\|y_{k-1}\right\|+\left|W_{k}\right|}{\xi_{0}\left\|F_{k-1}\right\|^{2}} \frac{\left\|F_{k}\right\|\left\|d_{k-1}\right\|}{\left\|F_{k}\right\|^{2}}\left\|F_{k}\right\| \\
& =\left\|F_{k}\right\|+2 \frac{\left\|F_{k}\right\|\left\|y_{k-1}\right\|+\left|W_{k}\right|}{\xi_{0}\left\|F_{k-1}\right\|^{2}}\left\|d_{k-1}\right\| \\
& \leq \kappa+\frac{4 m \kappa\left\|x_{k}-x_{k-1}\right\|}{\xi_{0} \theta^{2}}\left\|d_{k-1}\right\| .
\end{aligned}
$$

Now, from (3.6) there exist $c \in(0,1)$ such that

$$
\frac{4 m \kappa\left\|x_{k}-x_{k-1}\right\|}{\xi_{0} \theta^{2}}<c .
$$

Therefore, for $k>k_{0}$ we have

$$
\begin{aligned}
\left\|d_{k}\right\| & \leq \kappa+c\left\|d_{k-1}\right\| \\
& \leq \kappa\left(1+c+c^{2}+\ldots+c^{k-k_{0}+1}\right)\left\|d_{k_{0}}\right\| \\
& \leq \frac{\kappa}{1-c}+\left\|d_{k_{0}}\right\| .
\end{aligned}
$$


Setting $M=\left\{\left\|d_{1}\right\|,\left\|d_{2}\right\|, \ldots,\left\|d_{k_{0}}\right\|, \frac{\kappa}{1-c}+\left\|d_{k_{0}}\right\|\right\}$, we get (3.14). It also follows, in a similar way, that our direction is also bounded by the choice of $\beta_{k}^{2}$.

Theorem 3.4. Suppose that $\left\{x_{k}\right\}$ is generated using Algorithm 2.1. Then

$$
\liminf _{k \rightarrow \infty}\left\|F_{k}\right\|=0 .
$$

Proof. Suppose that (3.18) does not hold, that is, there exists a positive constant $\theta$ such that

$$
\left\|F_{k}\right\|>\theta, \quad \forall k \geq 0 .
$$

Observe that, from the sufficient descent condition (1.12) and the Cauchy-Schwarz inequality we have

$$
\left\|F_{k}\right\|\left\|d_{k}\right\| \geq-F_{k} d_{k} \geq\left\|F_{k}\right\|^{2} .
$$

Therefore, from (3.20) we get

$$
\left\|d_{k}\right\| \geq\left\|F_{k}\right\|>\theta>0 .
$$

This together with result in (3.8) implies that

$$
\lim _{k \rightarrow \infty} \alpha_{k}=0
$$

From the definition of the line search procedure, $\alpha_{k} \gamma^{-1}$ does not satisfy (2.14), i.e.,

$$
-F\left(x_{k}+\alpha_{k} \gamma^{-1} d_{k}\right)^{T} d_{k}<\delta \alpha_{k} \gamma^{-1}\left\|F\left(x_{k}+\alpha_{k} \gamma^{-1} d_{k}\right)\right\|\left\|d_{k}\right\|^{2} .
$$

It is therefore obvious from the boundedness of $\left\{x_{k}\right\}$ that $\left\{x_{k}\right\}$ has some accumulation point $\hat{x}$ and an infinite index set $Z_{1}$ such that $\lim _{k \in Z_{1}} x_{k}=\hat{x}$. It follows from (3.14) that $\left\{d_{k}\right\}_{k \in Z_{1}}$ is bounded as well. Therefore, there exist an infinite index set $Z_{2} \subset Z 1$ and some accumulation point $\hat{d}$ such that $\lim _{k \in Z_{2}} d_{k}=\hat{d}$. Now, if we take the limit in (3.23), we get

$$
-F(\hat{x})^{T} \hat{d} \leq 0 .
$$

Also taking the limit in sufficient descent condition (1.12), we get

$$
-F(\hat{x})^{T} \hat{d} \geq 0 .
$$

This gives rise to a contradiction, so (3.18) holds, and the proof is complete.

\section{NumericAl EXPERIMENT}

This section provide the numerical tests using the proposed Algorithm 2.1. The algorithm is coded in Matlab and comparison is provided in term efficiency with the NHZ derivative-free method [7] and the Self adaptive spectral conjugate gradient method for solving nonlinear monotone equations (SASCG) [19]. However, for the proposed algorithms we selected $\tau=1, \gamma=0.9, \delta=0.0001$ and $\xi_{0}=0.06$. Although for both the NHZ and SASCG methods, we implemented the default parameters used in the respective papers. In addition, iteration is terminated if $\left\|F\left(x_{k}\right)\right\| \leq 10^{-11}$ or the number of iteration is higher than 1000 on the following test problems:

Problem 4.1 ([21]). The precise description of the $F(x)$ function is described as

$$
F\left(x_{i}\right)=\exp \left(x_{i}\right)-1, \quad \text { for } \quad i=1,2,3, \ldots, n .
$$


Problem $4.2([19])$. The precise description of the function $F(x)$ is described as

$$
\begin{aligned}
F_{1}(x) & =h x_{1}+x_{2}-1, \\
F_{i}(x) & =x_{i-1}+h x_{i}+x_{i-1}-1, \quad \text { for } \quad i=2,3, \ldots, n-1, \quad h=2.5, \\
F_{n}(x) & =x_{n-1}+h x_{n}-1 .
\end{aligned}
$$

Problem 4.3 ([26]). The precise description of the function $F(x)$ is described as

$$
F\left(x_{i}\right)=x_{i}-\sin \left|x_{i}-1\right|, \quad \text { for } \quad i=1,2,3, \ldots, n .
$$

Problem 4.4 ([26]). The precise description of the function $F(x)$ is described as

$$
F\left(x_{i}\right)=2 x_{i}-\sin \left|x_{i}\right|, \quad \text { for } \quad i=1,2,3, \ldots, n .
$$

Problem 4.5 ([16]). The precise description of the function $F(x)$ is described as

$$
\begin{aligned}
F_{1}(x) & =x_{1}\left(x_{1}^{2}+x_{2}^{2}\right)-1, \\
F_{i}(x) & =x_{i}\left(x_{i-1}^{2}+2 x_{i}^{2}+x_{i+1}^{2}\right)-1, \quad \text { for } \quad i=2,3, \ldots, n-1, \\
F_{n}(x) & =x_{n}\left(x_{n-1}^{2}+x_{n}^{2}\right) .
\end{aligned}
$$

Problem 4.6 ([13]). The precise description of the function $F(x)$ is described as

$$
F_{i}(x)=x_{i}-\left(1-\frac{c}{2 n} \sum_{j=1}^{n} \frac{\mu_{i} x_{j}}{\mu_{i}+\mu_{j}}\right)^{-1} \quad, \quad \text { for } \quad i=1,2, \ldots, n, \quad \mu=\frac{i-0.5}{n}, \quad c=0.9 .
$$

Problem 4.7 ([28]). The precise description of the function $F(x)$ is described as

$$
\begin{aligned}
& F\left(x_{1}\right)=x_{1}-\exp \left(\frac{\cos \left(x_{1}+x_{2}\right)}{n+1}\right), \\
& F\left(x_{i}\right)=x_{i}-\exp \left(\frac{\cos \left(x_{i-1}+x_{i}+x_{i+1}\right)}{n+1}\right), \text { for } i=2,3, \ldots, n-1, \\
& F\left(x_{n}\right)=x_{n}-\exp \left(\frac{\cos \left(x_{n-1}+x_{n}\right)}{n+1}\right) .
\end{aligned}
$$

Problem $4.8([32,33])$. The precise description of the function $F(x)$ is described as

$$
F(x)=\left(\begin{array}{ccccc}
\frac{5}{2} & 1 & & & \\
1 & \frac{5}{2} & 1 & & \\
& \ddots & \ddots & \ddots & \\
& & \ddots & \ddots & 1 \\
& & & 1 & \frac{5}{2}
\end{array}\right)\left(\begin{array}{c}
x_{1} \\
x_{2} \\
\vdots \\
\\
x_{n}
\end{array}\right)+\left(\begin{array}{c}
1 \\
1 \\
\vdots \\
1
\end{array}\right) .
$$

Tables 1-4 showed the numerical efficiency of the proposed methods compared to the NHZ method [7] and the SASCG method [19]. In our comparison, ITER is set to represent the number of iterations, TIME for the CPU time in the second, FVL for the number of function evaluations, and NORM to indicate the norm of the function evaluation at the stopping point. On the following initial guesses, we considered eight test problems, namely, $x_{1}=(1,1, \ldots, 1), x_{2}=\left(1, \frac{1}{2}, \frac{1}{3}, \ldots, \frac{1}{n}\right), x_{3}=(0.1,0.1, \ldots, 0.1), x_{4}=\left(\frac{1}{n}, \frac{2}{n}, \ldots, 1\right), x_{5}=\left(1-\frac{1}{n}, 1-\frac{2}{n}, \ldots, 0\right)$, $x_{6}=(-1,-1, \ldots,-1), x_{7}=\left(n-\frac{1}{n}, n-\frac{2}{n}, \ldots, n-1\right)$ and $x_{8}=\left(\frac{1}{2}, 1, \frac{2}{3}, \ldots, \frac{2}{n}\right)$. 


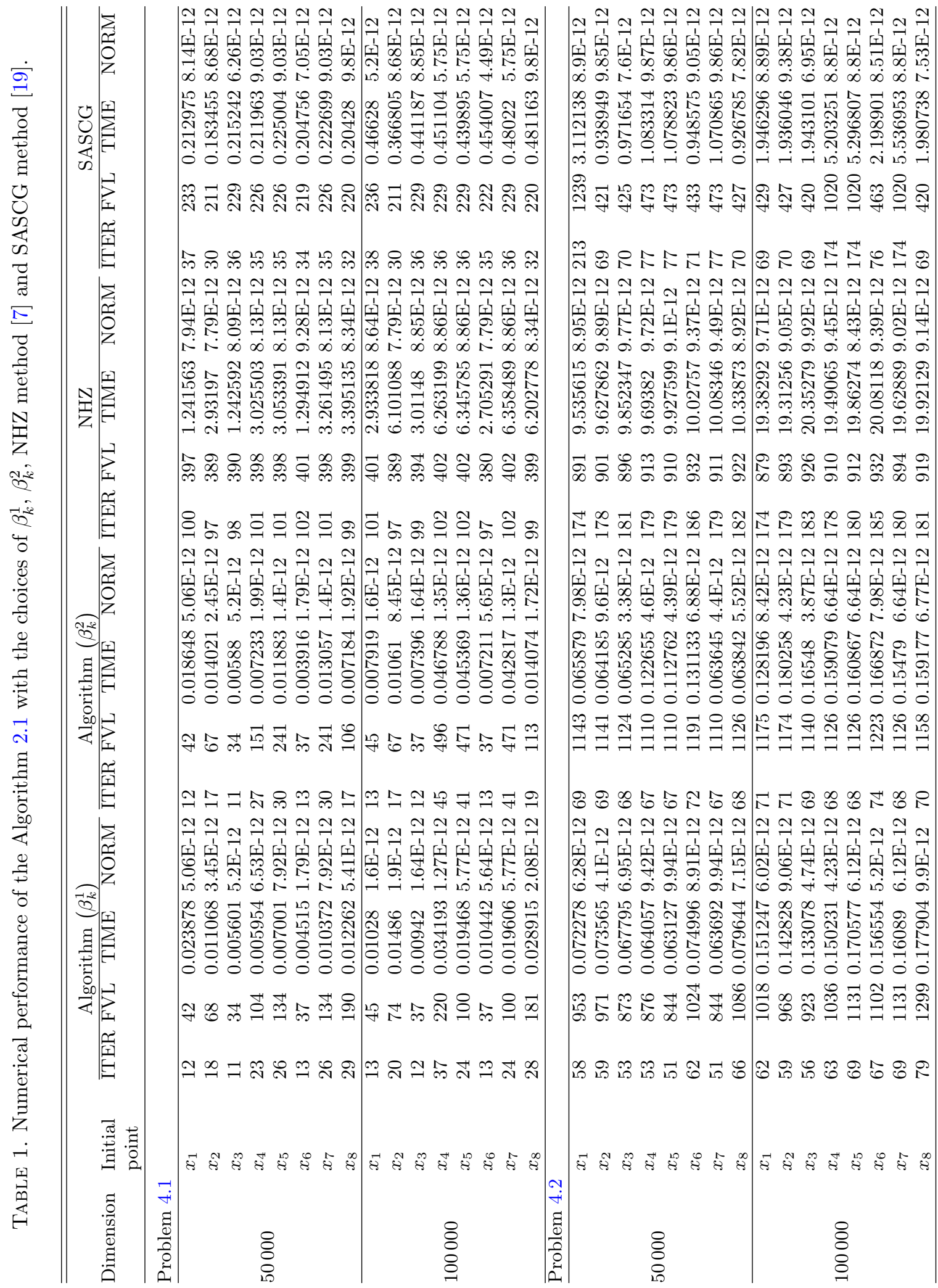




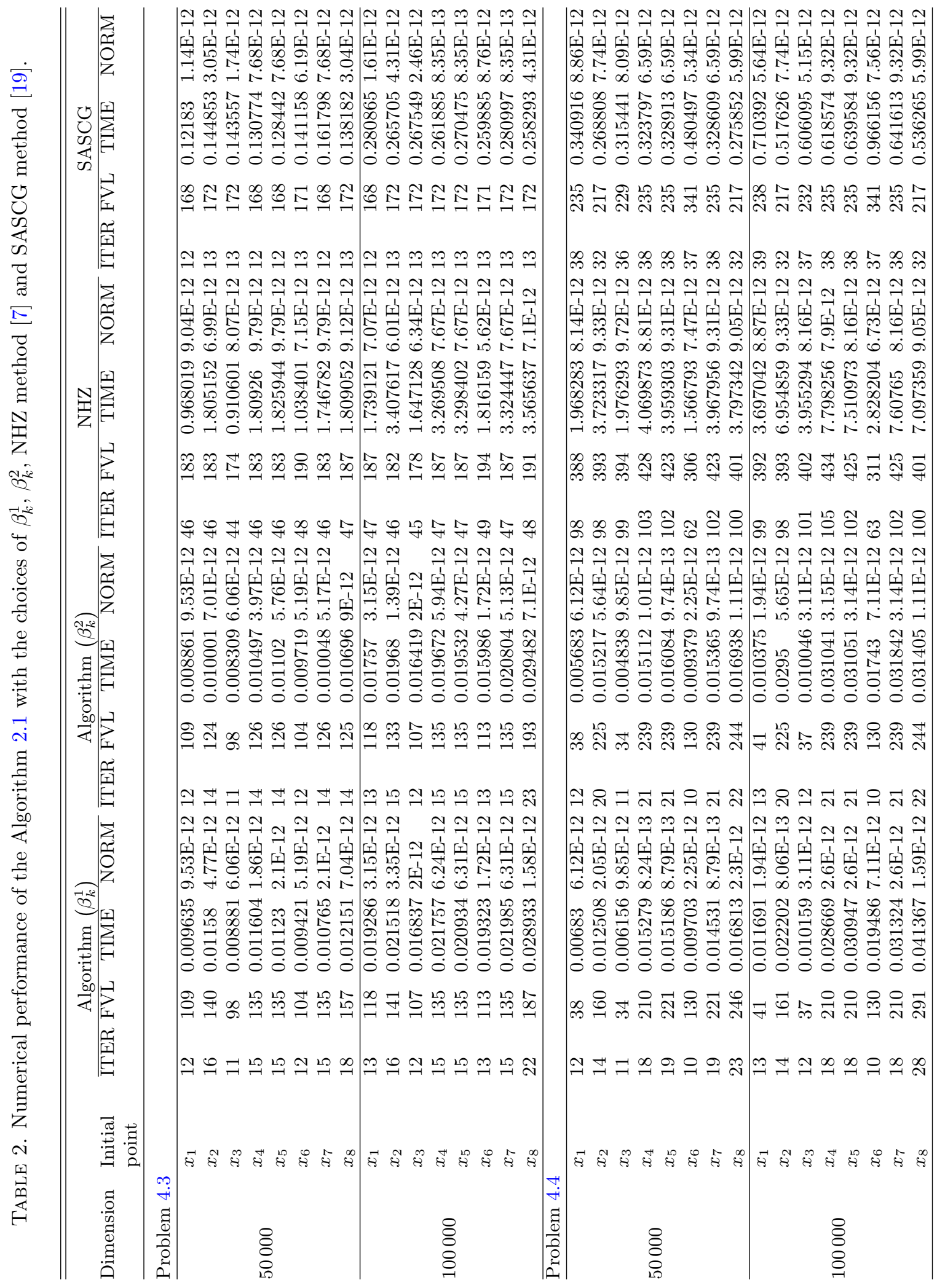




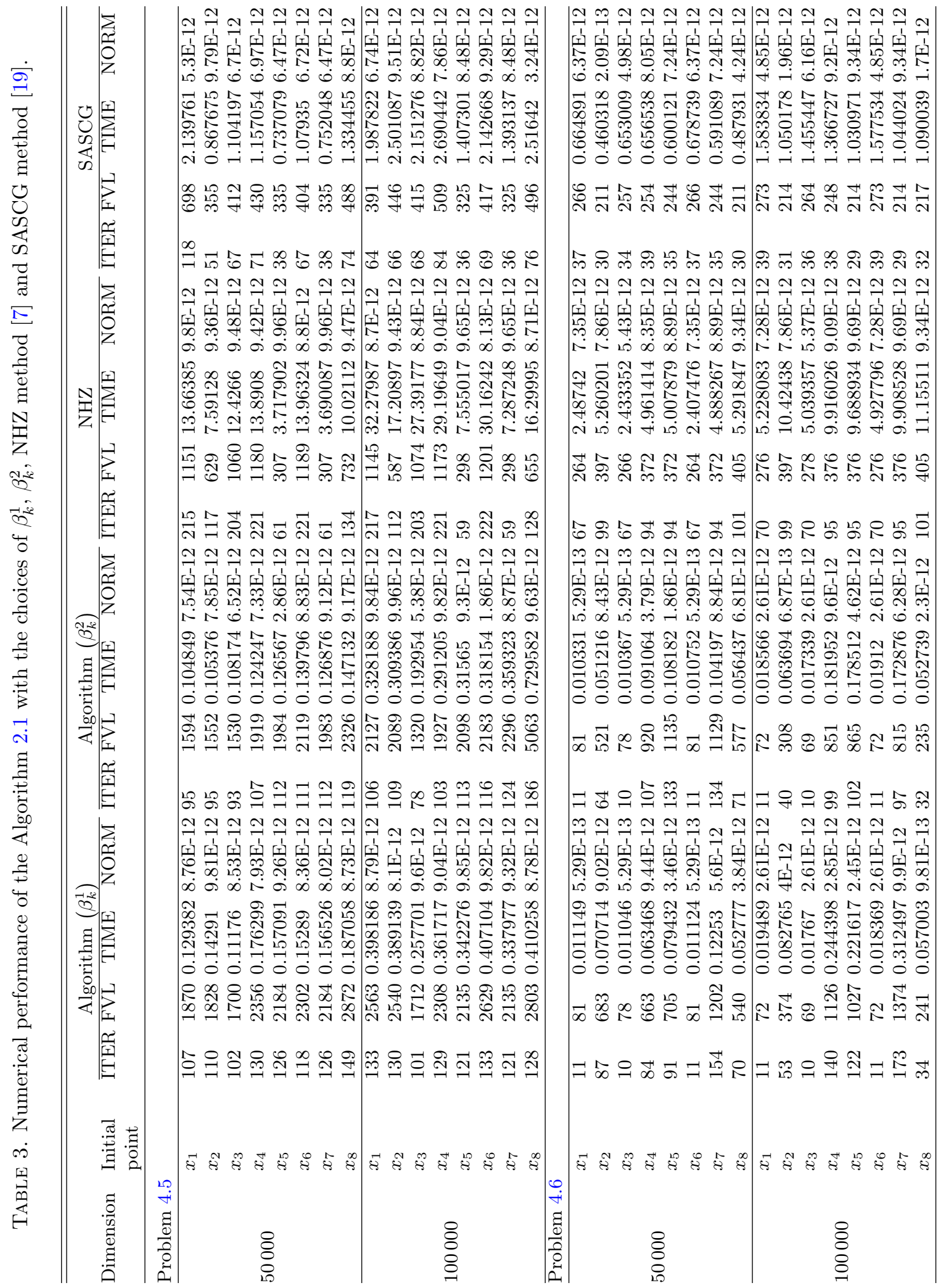




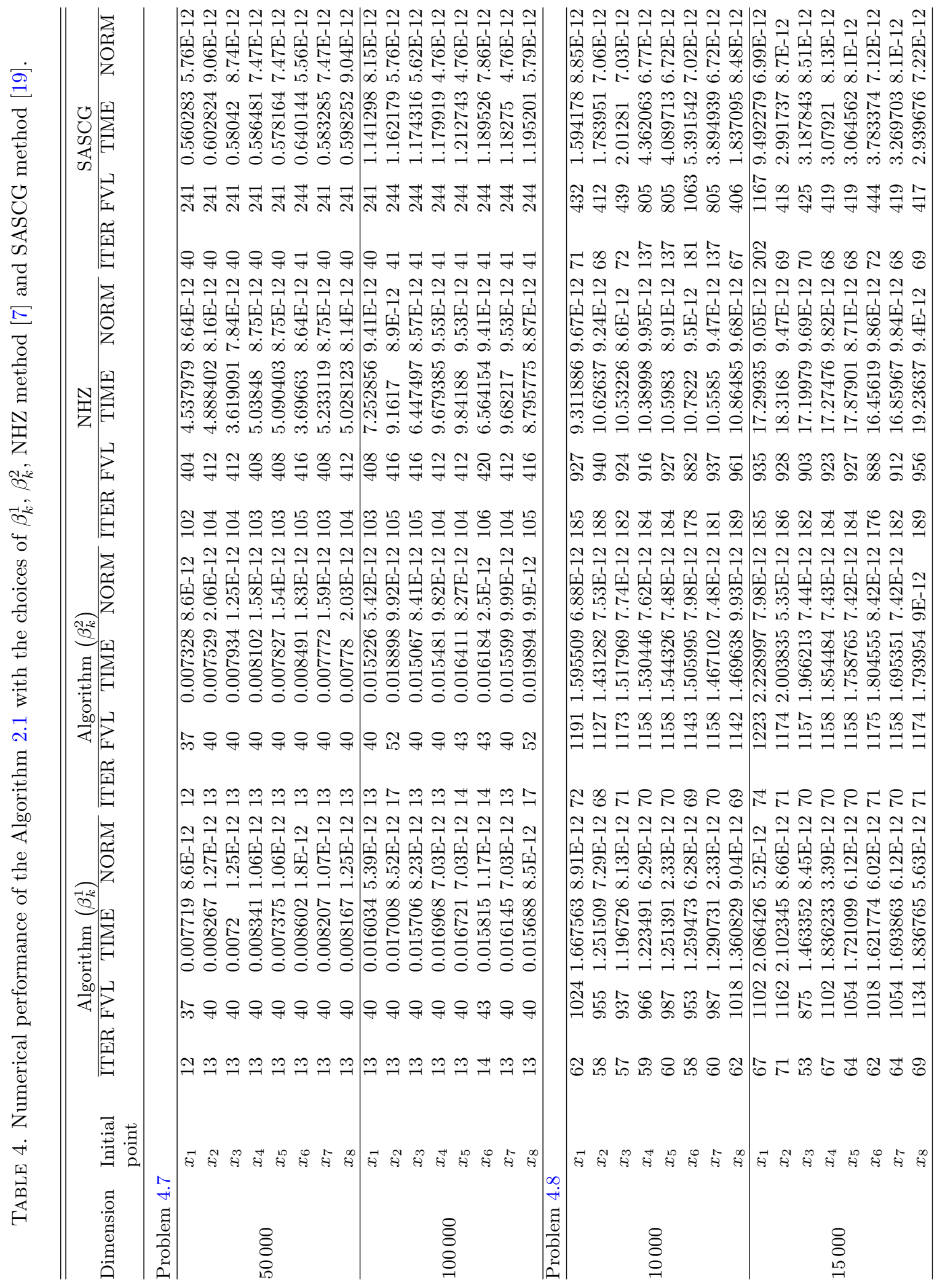




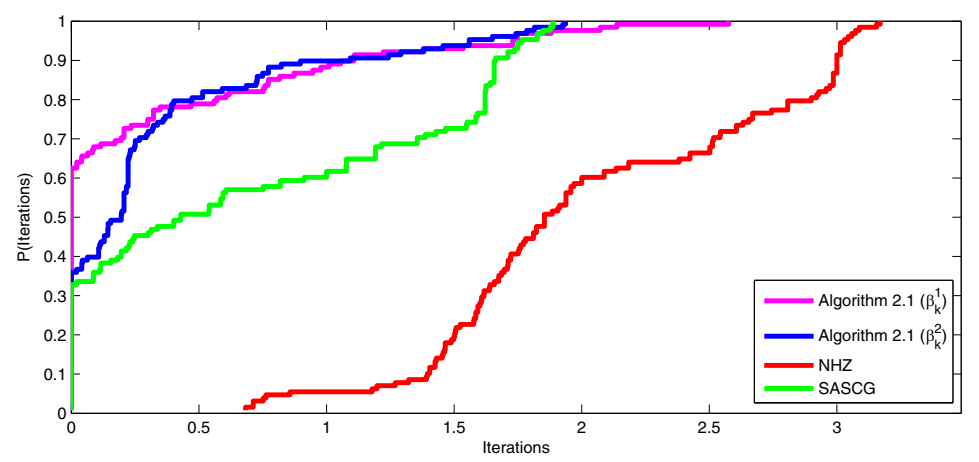

FIgURE 1. Performance of Algorithm 2.1 versus NHZ method [7] and SASCG method [19] (with respect to number of iteration).

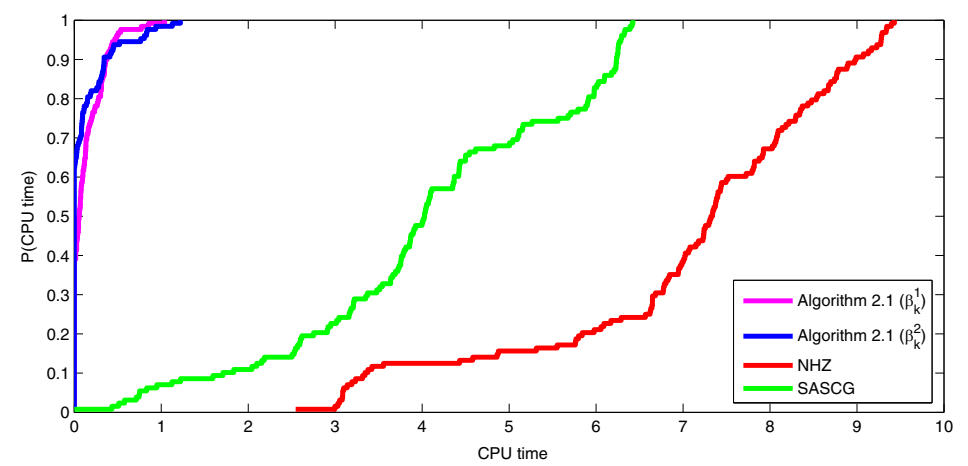

FiguRE 2. Performance of Algorithm 2.1 versus NHZ method [7] and SASCG method [19] (with respect to CPU time).

The Algorithm 2.1 with the choice $\beta_{k}^{1}$ has a relatively minimal number of iterations for the Table 1 compared to the Algorithm 2.1 with the choice of $\beta_{k}^{2}$, the NHZ method [7] and the SASCG method [19]. However, the Algorithm 2.1 with choice $\beta_{k}^{2}$ has a minimum number of the CPU time compared to the remaining methods. In addition, for Table 2, the Algorithm 2.1 with two choices of the $\beta_{k}$ has a minimal number of iterations compared to the other two methods. For the CPU time, the second-choice algorithm wins over the problems with almost $99 \%$.

In contrast, from Tables 3 and 4, our proposed algorithms also have fewer iterations and CPU time than the remaining methods, especially for Problems 4.7 and 4.8. Nevertheless, the SASCG method [19] has on average shown some impact on the minimum number of iterations for Problems 4.5 and 4.6. However, the overall performance in terms of less number of iterations and CPU time is based on our proposed methods with more than $70 \%$ for all the problems considered. However, with regard to the number of function evaluations, the two $\beta_{k}$ choices in our algorithm often compete with the NHZ method [7] and the SASCG method [19]. We also plotted the three figures to illustrate the performance of our methods using the performance profiles of Dolan and Moré [11]. It is remarkable to note that, based on the Dolan and Moré procedure, the top curve in the figure has advantages over the remaining curves (Figs. 1-3). 


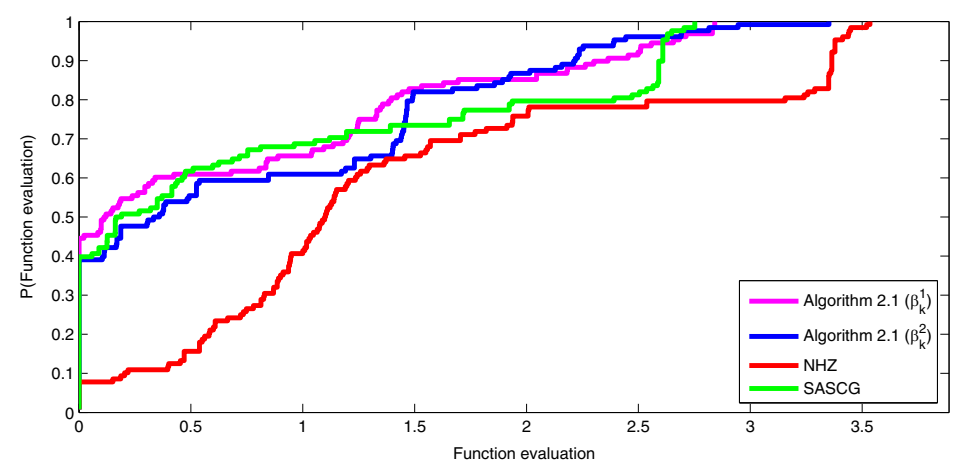

FIGURE 3. Performance of Algorithm 2.1 versus NHZ method [7] and SASCG method [19] (with respect to number of function evaluations).

\section{Conclusion}

We presented promising three-term CG-type methods to solve large-scale monotone nonlinear equations. Based on the idea of modifying the Dai-Liao conjugacy condition using the optimal choices of the non-negative parameter $t$, we suggested two new CG updates. However, to demonstrate the effectiveness of the suggested CG updates, we incorporated them into the Solodov and Svaiter projection techniques and solved monotone nonlinear equations. We proved the global convergence result of the proposed method and lastly used some test problems for the numerical efficacy of our methods compared to the NHZ Algorithm [7] and the SASCG method [19].

Acknowledgements. The first author is grateful to TWAS-CUI for the FR number award: 3240299486. We would like to thank the editor and all the reviewers for their valuable comments and suggestions.

\section{REFERENCES}

[1] N. Andrei, Open problems in nonlinear conjugate gradient algorithms for unconstrained optimization. Bull. Malays. Math. Sci. Soc. 34 (2011) 319-330.

[2] S. Babaie-Kafaki and R. Ghanbari, The Dai-Liao nonlinear conjugate gradient method with optimal parameter choices. Eur. J. Oper. Res. 234 (2014) 625-630.

[3] J. Barzilai and J.M. Borwein, Two point stepsize gradient methods. IMA J. Numer. Anal. 8 (1988) 141-148.

[4] W. Cheng, A PRP type method for systems of monotone equations. Math. Comput. Model. 50 (2009) 15-20.

[5] Y.H. Dai and L.Z. Liao, New conjugacy conditions and related nonlinear conjugate gradient methods. Appl. Math. Optim. 43 (2001) 87-101.

[6] Y.H. Dai and Y. Yuan, A nonlinear conjugate gradient method with a strong global convergence property. SIAM J. Optim. 10 (1999) 177-182.

[7] Z. Dai and H. Zhu, A modified Hestenes-Stiefel-type derivative-free method for large-scale nonlinear monotone equations. Mathematics 8 (2020) 168.

[8] Z.F. Dai, X. Chen and F. Wen, A modifed Perry's conjugate gradient method-based derivative-free method for solving largescale nonlinear monotone equations. Appl. Math. Comput. 270 (2015) 378-386.

[9] Z. Dai, H. Zhou, F. Wen and S. He, Efficient predictability of stock return volatility: the role of stock market implied volatility. North Am. J. Econ. Finance 52 (2020) 101174.

[10] J.E. Dennis and R.B. Schnabel, Numerical Methods for Unconstrained Optimization and Nonlinear Equations. Prentice-Hall, Englewood Cliffs, NJ (1983).

[11] E.D. Dolan and J.J. Moré, Benchmarking optimization software with performance profiles. Math. Program. 91 (2002) $201-213$.

[12] M. Figueiredo, R. Nowak and S.J. Wright, Gradient projection for sparse reconstruction, application to compressed sensing and other inverse problems. IEEE J. Sel. Top. Sign. Proces. 1 (2007) 586-597.

[13] P. Gao and C. He, An efficient three-term conjugate gradient method for nonlinear monotone equations with convex constraints. Calcolo 55 (2018) 53

[14] W.W. Hager and H. Zhang, A survey of nonlinear conjugate gradient methods. Pac. J. Optim. 2 (2006) 35-58. 
[15] M.R. Hestenes and E. Stiefel, Methods of conjugate gradients for solving linear systems. J. Res. Natl. Bur. Stand. 49 (1952) $409-436$.

[16] Y. Hu and Z. Wei, A modified Liu-Storey conjugate gradient projection algorithm for nonlinear monotone equations. Int. Math. Forum 9 (2014) 1767-1777.

[17] A.N. Iusem and M.V. Solodov, Newton-type methods with generalized distances for constrained optimization. Optimization 41 (1997) 257-278.

[18] H. Kobayashi, Y. Narushima and H. Yabe, Descent three-term conjugate gradient methods based on secant conditions for unconstrained optimization. Optim. Methods Softw. 32 (2017) 1313-1329.

[19] M. Koorapetse and P. Kaelo, Self adaptive spectral conjugate gradient method for solving nonlinear monotone equations. J. Egypt. Math. Soc. 28 (2020) 4.

[20] Q. Li and D.H. Li, A class of derivative-free methods for large-scale nonlinear monotone equations. IMA J. Numer. Anal. 31 (2011) $1625-1635$.

[21] J. Liu and S. Li, Spectral DY-type projection method for nonlinear monotone systems of equations. J. Comput. Math. 33 (2015) 341-354.

[22] K. Meintjes and A.P. Morgan, A methodology for solving chemical equilibrium systems. Appl. Math. Comput. 22 (1987) $333-361$.

[23] Y. Narushima and H. Yabe, A survey of sufficient descent conjugate gradient methods for unconstrained optimization. SUT J. Math. 50 (2014) 167-203.

[24] Y. Narushima, H. Yabe and J.A. Ford, A three-term conjugate gradient method with sufficient descent property for unconstrained optimization. SIAM J. Optim. 21 (2011) 212-230.

[25] J.M. Ortega and W.C. Rheinboldt, Iterative Solution of Nonlinear Equations in Several Variables. SIAM, Philadelphia, PA (1970).

[26] J. Sabi'u, A. Shah and M.Y. Waziri, Two optimal Hager-Zhang conjugate gradient methods for solving monotone nonlinear equations. Appl. Numer. Math. 153 (2020) 217-233.

[27] M.V. Solodov and B.F. Svaiter, A globally convergent inexact Newton method for systems of monotone equations. Reformulation: Nonsmooth, Piecewise Smooth, Semismooth and Smoothing Methods, edited by M. Fukushima and L. Qi. In Vol. 22 of Applied Optimization. Springer (1998) 355-369.

[28] S. Wang and H. Guan, A scaled conjugate gradient method for solving monotone nonlinear equations with convex constraints. J. Appl. Math. 2013 (2013) 286486.

[29] M.Y. Waziri, K.A. Hungu and J. Sabi'u, Descent Perry conjugate gradient methods for systems of monotone nonlinear equations. Numer. Algorithms $\mathbf{8 5}$ (2020) 763-785.

[30] L. Zhang and W. Zhou, Spectral gradient projection method for solving nonlinear monotone equations. J. Comput. Appl. Math. 196 (2006) 478-484.

[31] Y.B. Zhao and D.H. Li, Monotonicity of fixed point and normal mapping associated with variational inequality and its application. SIAM J. Optim. 4 (2001) 962-973.

[32] W.J. Zhou and D.H. Li, Limited memory BFGS method for nonlinear monotone equations. J. Comput. Math. 25 (2007) 89-96.

[33] W.J. Zhou and D.H. Li, A globally convergent BFGS method for nonlinear monotone equations without any merit functions. Math. Comput. 77 (2008) 2231-2240.

[34] G. Zhou and K.C. Toh, Superlinear convergence of a Newton-type algorithm for monotone equations. J. Optim. Theory App. 125 (2005) 205-221. 